\title{
Correction to: The arithmetic of Carmichael quotients
}

\author{
Min Sha ${ }^{1}$
}

Published online: 30 November 2017

(C) Akadémiai Kiadó, Budapest, Hungary 2017

\begin{abstract}
The statement of Proposition 4.3 in the published paper is not correct. Here we change the statement and give a complete proof.
\end{abstract}

\section{Erratum to: Period Math Hung (2015) 71:11-23 https://doi.org/10.1007/s10998-014-0079-3}

\section{Replacement of [3, Proposition 4.3]}

We first illustrate a counter-example for [3, Proposition 4.3]. Take $m=273=3 \times 7 \times$ 13. Using the notation in [3, Proposition 4.3], we have $d=3$ and $d^{\prime}=1$. That is, the homomorphism $\phi_{m}$ defined there is surjective. However, for any positive integer $a$ coprime to $m, C_{m}(a)$ is divisible by 3 , because $6 \mid \lambda(m)$ and then $9 \mid a^{\lambda(m)}-1$. This leads to a contradiction.

Proposition 4.3 and its proof in [3] should be replaced by Proposition 1.1 below. Fortunately, this does not affect other results and arguments in [3], although Proposition 4.3 in [3] was quoted several times there.

Assume that positive integer $m$ has the prime factorization $m=p_{1}^{r_{1}} \cdots p_{k}^{r_{k}}$. In [1, Proposition 4.4], the Euler quotient has been used to define a homomorphism from $\left(\mathbb{Z} / m^{2} \mathbb{Z}\right)^{*}$ to $(\mathbb{Z} / m \mathbb{Z},+)$, whose image is $d \mathbb{Z} / m \mathbb{Z}$, where

$$
d=\prod_{i=1}^{k} d_{i} \quad \text { and } \quad d_{i}=\left\{\begin{array}{l}
\operatorname{gcd}\left(p_{i}^{r_{i}}, 2 \varphi(m) / \varphi\left(p_{i}^{r_{i}}\right)\right) \text { if } p_{i}=2 \text { and } r_{i} \geq 2, \\
\operatorname{gcd}\left(p_{i}^{r_{i}}, \varphi(m) / \varphi\left(p_{i}^{r_{i}}\right)\right) \text { otherwise. }
\end{array}\right.
$$

In fact, the above $d, d_{i}$ are equivalent to those $d, d_{i}$ defined in [3], respectively.

The online version of the original article can be found under https://doi.org/10.1007/s10998-014-0079-3.

Min Sha

shamin2010@gmail.com

1 Department of Computing, Macquarie University, Sydney, NSW 2109, Australia 
By [3, Proposition 2.2 (2)], the Carmichael quotient $C_{m}(x)$ induces a homomorphism

$$
\phi_{m}:\left(\mathbb{Z} / m^{2} \mathbb{Z}\right)^{*} \rightarrow(\mathbb{Z} / m \mathbb{Z},+), x \mapsto C_{m}(x),
$$

where $C_{m}(x)=\left(x^{\lambda(m)}-1\right) / m$ and $\lambda(m)$ is the Carmichael function.

Proposition 1.1 Let $m=p_{1}^{r_{1}} \cdots p_{k}^{r_{k}}$ be the prime factorization of $m \geq 2$. For $1 \leq i \leq k$, put

$$
d_{i}^{\prime}= \begin{cases}\operatorname{gcd}\left(p_{i}^{r_{i}}, 2 \lambda(m) / \lambda\left(p_{i}^{r_{i}}\right)\right) & \text { if } p_{i}=2 \text { and } r_{i}=2, \\ \operatorname{gcd}\left(p_{i}^{r_{i}}, \lambda(m) / \lambda\left(p_{i}^{r_{i}}\right)\right) & \text { otherwise. }\end{cases}
$$

Let $d^{\prime}=\prod_{i=1}^{k} d_{i}^{\prime}$. Then the image of the homomorphism $\phi_{m}$ is $d^{\prime} \mathbb{Z} / m \mathbb{Z}$.

Proof We show the desired result case by case.

(I) First we prove the result for the case $k=1$, that is $m=p^{r}$, where $p$ is a prime and $r$ is a positive integer.

Suppose that $p=2$. If $r=2$, then $C_{m}(3)=2$, and for any positive integer $n$ we have $C_{m}(2 n+1)=n(n+1)$, which is even, so the image of $\phi_{m}$ is $2 \mathbb{Z} / m \mathbb{Z}$. On the other hand, if $r=1$ or $r \geq 3$, since $C_{2}(3)=1$ and $C_{8}(3)=1$, by using [3, Proposition 2.8] we see that $C_{m}(3)$ is an odd integer, so the image of $\phi_{m}$ is $\mathbb{Z} / m \mathbb{Z}$.

Now, assume that $p>2$. Note that $C_{p}(p+1) \equiv-1(\bmod p)$, by [3, Proposition 2.8] we have $C_{m}(p+1) \equiv-1(\bmod p)$, which implies that $p \nmid C_{m}(p+1)$. Thus, there exists a positive integer $n$ such that $n C_{m}(p+1) \equiv 1(\bmod m)$. Then, by [3, Proposition 2.2 (1)] we deduce that $C_{m}\left((p+1)^{n}\right) \equiv 1(\bmod m)$. So, the image of $\phi_{m}$ is $\mathbb{Z} / m \mathbb{Z}$.

(II) To complete the proof, we prove the result when $k \geq 2$.

For simplicity, denote $m_{i}=m / p_{i}^{r_{i}}$ and $n_{i}=\lambda(m) / \lambda\left(p_{i}^{r_{i}}\right)$ for each $1 \leq i \leq k$, and then let $m_{i}^{\prime}$ be an integer such that $m_{i}^{2} m_{i}^{\prime} \equiv 1\left(\bmod p_{i}^{r_{i}}\right)$. By [3, Proposition 2.7], we have

$$
C_{m}(a) \equiv \sum_{i=1}^{k} m_{i} m_{i}^{\prime} n_{i} C_{p_{i}^{r_{i}}}(a) \quad(\bmod m)
$$

So, for each $1 \leq i \leq k, C_{m}(a) \equiv m_{i} m_{i}^{\prime} n_{i} C_{p_{i}^{r_{i}}}(a)\left(\bmod p_{i}^{r_{i}}\right)$. If $p_{i}=2$ and $r_{i}=2$, note that for any odd integer $a>1, C_{4}(a)$ is even, then we see that $d_{i}^{\prime} \mid n_{i} C_{p_{i}} r_{i}(a)$, and thus $d_{i}^{\prime} \mid C_{m}(a)$. Otherwise if $p_{i}>2$ or $r_{i} \neq 2$, then $d_{i}^{\prime} \mid n_{i}$, and so $d_{i}^{\prime} \mid C_{m}(a)$. Hence, we have $d^{\prime} \mid C_{m}(a)$ for any integer $a$ coprime to $m$.

Let $b=\operatorname{gcd}\left(m, m_{1} m_{1}^{\prime} n_{1}, \ldots, m_{k} m_{k}^{\prime} n_{k}\right)$. Then, there exist integers $X_{1}, \ldots, X_{k}$ such that

$$
b \equiv \sum_{i=1}^{k} m_{i} m_{i}^{\prime} n_{i} X_{i} \quad(\bmod m) .
$$

If we denote $b_{i}=\operatorname{gcd}\left(p_{i}^{r_{i}}, m_{i} m_{i}^{\prime} n_{i}\right)$ for each $1 \leq i \leq k$, then $b=\prod_{i=1}^{k} b_{i}$; here, we remark that $b_{i}=\operatorname{gcd}\left(p_{i}^{r_{i}}, n_{i}\right)$. It is easy to see that for each $1 \leq i \leq k$, if $p_{i}>2$ or $r_{i} \neq 2$, we have $d_{i}^{\prime}=b_{i}$. Further, when $p_{i}=2$ and $r_{i}=2, d_{i}^{\prime}=2 b_{i}$ if $8 \nmid \lambda\left(2 p_{1} \ldots p_{k}\right)$, and $d_{i}^{\prime}=b_{i}$ otherwise.

We now have three cases for $m$ :

(i) There exists $1 \leq j \leq k$ such that $p_{j}=2, r_{j}=2$ and

$$
8 \nmid \lambda\left(2 p_{1} \ldots p_{k}\right) .
$$


(ii) There exists $1 \leq j \leq k$ such that $p_{j}=2, r_{j}=2$ and

$$
8 \mid \lambda\left(2 p_{1} \ldots p_{k}\right) \text {. }
$$

(iii) All the other cases.

Clearly, in Cases (ii) and (iii) we have $d^{\prime}=b$, and in Case (i) $d^{\prime}=2 b$.

According to (I), there exist integers $a_{i}$ with $p_{i} \nmid a_{i}$ for $1 \leq i \leq k$ defined by

$$
C_{p_{i}^{r_{i}}}\left(a_{i}\right) \equiv\left\{\begin{array}{ll}
2 X_{i} & \text { in Case (i), } \\
X_{i} & \text { in Case (iii), } \\
X_{i} & \text { in Case (ii) and } i \neq j, \\
0 & \text { in Case (ii) and } i=j .
\end{array}\left(\bmod p_{i}^{r_{i}}\right)\right.
$$

By the Chinese Remainder Theorem, we can choose a positive integer $a$ such that $a \equiv a_{i}$ $\left(\bmod p_{i}^{2 r_{i}}\right)$. So, by [3, Proposition $\left.2.2(2)\right]$ we have $C_{p_{i}^{r_{i}}}(a) \equiv C_{p_{i}^{r_{i}}}\left(a_{i}\right)\left(\bmod p_{i}^{r_{i}}\right)$. Then, combining with (1.3) and the relation between $b$ and $d^{\prime}$, we obtain $m_{i} m_{i}^{\prime} n_{i} C_{p_{i}^{r_{i}}}(a) \equiv d^{\prime}$ $\left(\bmod p_{i}^{r_{i}}\right)$ for each $1 \leq i \leq k$ in all the three cases. Finally, using (1.2) we have $C_{m}(a) \equiv d^{\prime}$ $(\bmod m)$, which completes the proof.

Comparing (1.1) with Proposition 1.1, we have $d^{\prime} \mid d$. Moreover, by [3, Proposition 2.1] we get

$$
\frac{\varphi(m)}{\lambda(m)} d^{\prime} \mathbb{Z} / m \mathbb{Z}=d \mathbb{Z} / m \mathbb{Z}
$$

which implies that $\operatorname{gcd}\left(\frac{\varphi(m)}{\lambda(m)} d^{\prime}, m\right)=d$.

\section{Another error}

We take this opportunity to correct another error. In the proof of [3, Lemma 3.4], the last identity " $\equiv \ell n^{-1} 2^{r-2}$ " may be not true, and it should be deleted. Because by using $n^{2^{r-2}} \equiv 1$ $\left(\bmod 2^{r}\right)$, we only know that $\left(n^{2^{r-2}}+1\right) / 2$ is an odd integer, which may be not congruent to 1 modulo $2^{r}$. Clearly, this error does not change the result there.

Acknowledgements The author wants to thank the anonymous referee of his joint paper [2] for pointing out the error in [3, Proposition 4.3] and giving the counter-example.

\section{References}

1. T. Agoh, K. Dilcher, L. Skula, Fermat quotients for composite moduli. J. Number Theory 66, 29-50 (1997)

2. F. Luca, M. Sha, I.E. Shparlinski, On two functions arising in the study of the Euler and Carmichael quotients. Colloq. Math. 149, 179-192 (2017)

3. M. Sha, The arithmetic of Carmichael quotients. Period. Math. Hung. 71, 11-23 (2015) 